Valerie Nesset

University at Buffalo (SUNY), Buffalo, NY, U.S.A.

\title{
Researching the Researchers: Gathering Data on Academics' Use of Technology
}

\begin{abstract}
Résumé:
Bonded design, a participatory design methodology developed by information science researchers, is used as the framework for a university-wide initiative, the faculty IT liaison program, where faculty members and IT professionals work together as peers in design teams to examine and assess technologies. Bonded design met the program criteria: a limited and finite number of design sessions, opportunities to analyse data in situ to inform an iterative design process, and a framework to help two disparate groups (users and designers) to interact and collaborate with one another to generate innovative ideas for designing more userfriendly technologies.
\end{abstract}

\section{Introduction}

In the fast-paced $21^{\text {st }}$ century digital environment, it is a well-known fact that in higher education faculty members will be required to integrate new technologies into their research and especially teaching on a regular basis. Yet, while some faculty may be very comfortable using technology, others struggle. To address this, a project has been initiated in a large research university in New York State to bring faculty and IT staff together in participatory design teams to interact with existing campus-wide technologies and to share expertise. The faculty IT liaison program uses as its framework the bonded design methodology created to design technology in intergenerational teams (Large and Nesset, 2009; Large, Nesset, Beheshti, and Bowler, 2006, 2007). Bonded design brings users and designers of technology together in a shared experience within a participatory design team to learn from each other and bond in an iterative process of discovery through low-tech hands-on prototyping with the purpose of designing technologies that not only better serve users' needs, but also could not be designed by each group alone. The program consists of three stages that build upon each other, starting with a university-wide technology needs assessment which informs the second stage of participatory design sessions which in turn inform the final assessment and implementation stage. To date, the program is in the second stage.

\section{Conceptual Framework - Participatory Design}

The concept of participatory design originated in Scandinavian countries in response to the observation that workers were best able to improve their workplaces (Carmel, Whitaker, and George, 1993). Soon, participatory design's highly iterative and inclusive methods were introduced into technology design in the hopes that by including users in the process alongside experienced designers in such intrinsic roles as peer co-designer would result in the design of technologies that better meet users' needs. Furthermore, participatory design's use of two main techniques, metaphor and modeling to implement its two main principles, "mutual reciprocal learning" and "design by doing" where users and designers teach each other about work practices and technological possibilities using such techniques as interactive experimentation 
and hands-on design make it an effective tool to unite these two potentially disparate groups (Carmel, Whitaker, and George, 1993; Muller and Kuhn, 1993). Indeed, with the recent emphasis on user experience (UX) design (e.g., Gibbons, 2016; McLaughlin, 2015) it behooves designers of IT, before the technology is placed on the market, to gather and analyse data revealing the "technology hacks" users employ to discover affordances in the technologies that were not part of the original design. Participatory design methods, many of which mirror those of UX in involving users in all aspects of the process, offer an effective way to collect and analyse these data.

Several participatory design approaches were considered for the project: Bonded Design (Large and Nesset, 2009; Large, Nesset, Beheshti, and Bowler, 2006, 2007), Contextual Design (Beyer and Holtsblatt, 1999), Cooperative Inquiry (Druin, 1999; Guha, Druin, Chipman, et al., 2004), Informant Design (Scaife and Rogers, 1999), and Learner-Centered Design (Guzdial, 2016; Soloway, Guzdial, and Hay, 1994). To facilitate decision-making as to which approach would be the most appropriate for the aims of the faculty IT liaison program it was decided that any methodology needed to provide opportunities to be continually informed by data, have a flexible structure, have a finite timeline spanning no more than a few months, include several participatory design features from a variety of models, encourage diverse team membership, and be scalable to broader contexts. The bonded design methodology best met these criteria.

\section{Methodology}

Using the bonded design methodology as a guideline, the faculty IT liaison program is planned in three sequential stages: 1) a needs assessment of the greater user (faculty) population; 2) participatory design sessions with teams comprised of faculty (user) representatives from different academic units and IT professionals; and 3) assessment of the design outcomes to inform implementation. Assessment of the design process forms the research aspect of the program to ascertain whether the bonded design methodology is replicable, scalable, and transferable to different contexts.

\subsection{Stage 1: Needs Assessment}

The needs assessment is crucial to bonded design to determine the needs of the larger user group and to help inform and focus the design session planning process. The needs assessment for this project took the form of the first-ever university-wide IT survey of tenure-track, clinical, and adjunct faculty members. (Interestingly, while students attending the university are regularly polled on their technology uses and preferences this was the first time that faculty had been surveyed in such a manner.) The survey, administered in spring 2017, was created to determine faculty's use of university-owned and personal computer hardware, the age and model of their most-used and preferred hardware, smartphone carriers and use, and instructional technology use. The survey was designed by a steering committee comprised of the university's Chief Information Officer, the information science researcher, and representatives from IT and instructional facilities staff. The questions were subsequently reviewed by members of the university's faculty senate IT committee who provided suggestions regarding additional content, wording, and organization.

\subsection{Stage 2: The Design Sessions}

The original bonded design methodology consists of a maximum of 12 design sessions in which six specific design techniques are used by designers and users to collaborate within a team to create several low-tech prototypes of a specific technology, with the goal of gaining consensus on one final prototype (Figure 1). 


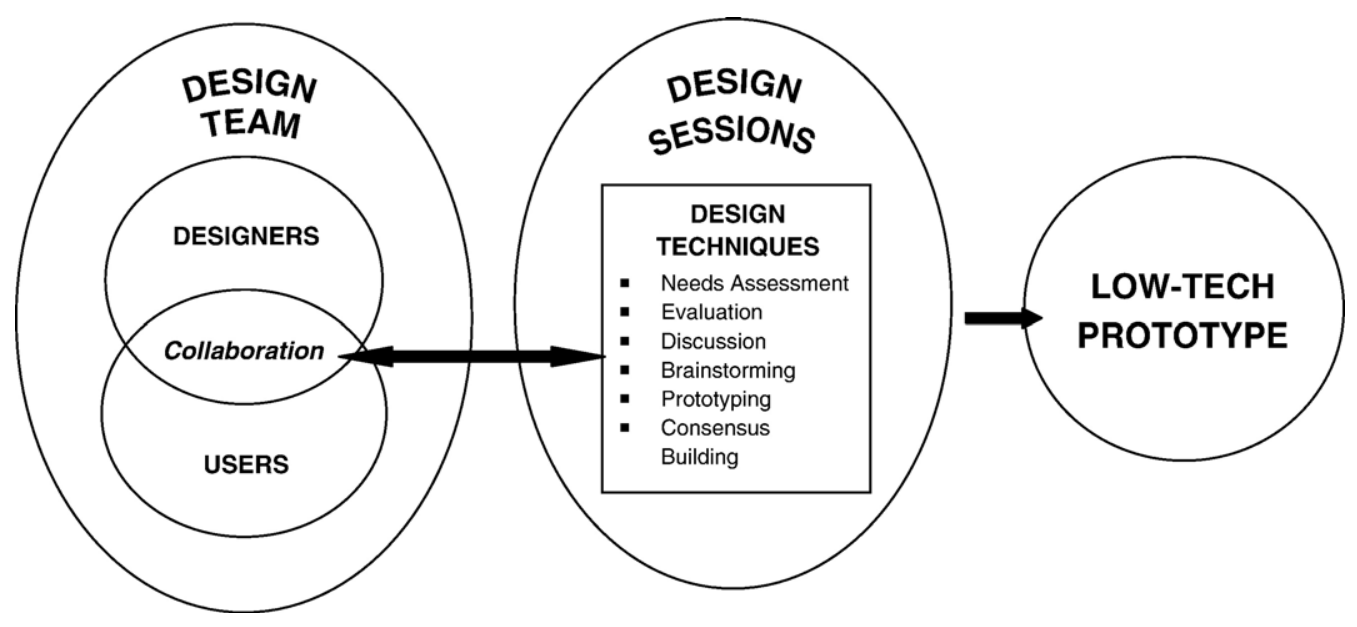

Fig 1. The Bonded Design Model (Large, et al., 2006)

When planning the faculty IT liaison program sessions, it was decided to begin with evaluation as the needs assessment had already been done.

Recruiting Participants - Bonded design provides no directives regarding recruitment of participants for the design teams from a large pool of users. Moreover, for the current project, for purposes of diversity and equity and to ensure the generation of representative data that could be used for future faculty-informed IT decisions by senior IT administrators, it was important for each team to be comprised of faculty representatives from different academic units. However, two major issues arose with this plan: How to recruit such representative participants and ensure that that they were "typical" technology users and not experts. Thus, the idea of the faculty IT liaison program was born. The program steering/planning committee reviewed research on effective groups (e.g., Johnson and Johnson, 1992) and decided that an application process would help with the faculty participant selection process. The online application form distributed via faculty email listserv briefly explained the purpose of the design sessions, outlined basic requirements of participation and a broad timeline, and asked questions regarding familiarity with technology, the university technologies the faculty member uses and for what purposes, and an open-ended question asking why the faculty member was interested in participating.

Data Collection Instruments - To better investigate the efficacy of the design team process as a means of gaining greater understanding of faculty's unique IT needs, data are being collected using a variety of methods: Participant observation where the researcher, through direct interaction with the participants essentially becomes a data collection instrument (Guba and Lincoln, 1985; Miles and Huberman, 1994); a pre- and post-questionnaire to allow for exploration of and comparison between the team members' expectations of the process and their perceptions of the process after its completion; one-on-one interviews with participants to gain further insights into the participants' perspectives than could be attained through the questionnaires (Patton, 2002); and videotaping of design sessions for later analysis to investigate actual behaviors to be compared with the participants' perceptions of the process.

Design Techniques - In bonded design, informed by the needs assessment, the five remaining design techniques (evaluation, discussion, brainstorming, prototyping, and consensus building) centre around the technologies identified for investigation. For this first study, the technologies were chosen by the faculty 
participants during two planning meetings. They include various aspects of the course management system (e.g., student grading center, course tools), email, and cloud storage solutions. As part of evaluation, team members will embark on activities such as drawing mental models of a technology or creating concept maps of what they think are the most important elements. These artifacts serve as a starting point for team discussion about the different interpretations of the technology followed by brainstorming sessions where each idea is considered equal and documented. As discovered in the bonded design studies with young students, it is during these non-judgmental brainstorming sessions where all team members are free to voice their thoughts that innovative and often surprising potential uses of a technology are discovered (Large and Nesset, 2009; Large, Nesset, Beheshti, and Bowler, 2007). After brainstorming is finished and the ideas discussed, covering the advantages, disadvantages, and feasibility of each, team members will start the hands-on iterative design process that involves strong collaboration and the frequent revisiting and revising of draft prototypes, in this case, modifications to the technologies and/or to existing instructions or protocols.

\subsection{Phase 3: Assessment}

While assessment is ongoing throughout the design sessions, consisting of evaluation and refinement of the protocols and technology modifications (the 'hacks') created by the design teams, consistent with action research (Wilson, 1980) the analysis and assessment of the prototypes performed by the program steering committee will be shared with the team members to ensure accuracy and solicit further feedback. Additionally, once the sessions end, each faculty liaison team member together with an IT professional team member together will hold at least one technology training session in their home academic units on what they learned during the program. Thus, the research takes on a direct-action component, benefiting participants and the wider university community.

\section{Conclusion}

The authors chose bonded design as a feasible methodology for the faculty IT liaison project as it is a context where time is a critical factor, collected data is analyzed in situ to inform an iterative process, and where two disparate groups of people with varying areas of expertise need to interact with one another to construct a tangible deliverable. The analysis and assessment of the data generated and of the bonded design methodology as a framework to guide the design sessions not only will test the premise made by the developers that it can be used to unite people with disparate expertise in a shared experience to generate ideas and innovations that would not occur to more homogenous groups, but also address the question of how information science theoretical frameworks, research methodologies, and approaches can inform and contribute to the study of data.

\section{Reference List:}

Beyer, Hugh and Karen Holtzblatt. 1999. "Contextual design.” ACM Interactions 6:32-42.

Carmel, Erran, Randall D. Whitaker, and Joey F. George. 1993. "PD and joint application design: A transatlantic comparison." Communications of the ACM 36(4):40-48

Druin, Allison. 1999 "Cooperative inquiry: Developing new technologies for children with children." in Proceedings of the SIGCHI conference on human factors in computing systems. ACM Press New York, pp 592-599

Gibbons, Sarah. 2016. "Design thinking 101.” Nielsen Norman Group. Retrieved from: https://www.nngroup.com/articles/design-thinking/. 
Guzdial, Mark. 2016. Learner-centered design of computing education: Research on computing for everyone. San Rafael, CA: Morgan \& Claypool Publishers.

Johnson, David W. and Roger T. Johnson. 1992. Positive interdependence: key to effective cooperation. Chapter 8 in Rahell Hertz-Lazarowitz and Norman Miller (Eds.), Interaction in cooperative groups: Theoretical anatomy of group learning. Cambridge: Cambridge University Press.

Large, Andrew and Valerie Nesset. 2009. "Bonded Design." Chapter 64 in Encyclopedia of Information Science and Technology, $2^{\text {nd }}$ Edition. Hershey PA: Information Science Reference.

Large, Andrew, Valerie Nesset, Jamshid Beheshti and Leanne Bowler. 2006. “'Bonded Design': A novel approach to intergenerational information technology design." Library and Information Science Research 28: $64-82$.

Large, Andrew, Valerie Nesset, Jamshid Beheshti, and Leanne Bowler. 2007. "Bonded Design: A methodology for designing with children." Chapter 4 in Advances in Universal Web Design and Evaluation: Research, Trends and Opportunities. Hershey PA: Idea Group.

Lincoln, Yvonna S. and Egon Guba. 1985. Naturalistic Inquiry. Newbury Park, CA: Sage Publications.

McLaughlin, Jean E. 2015. "Focus on user experience: Moving from a library-centric point of view." Internet Reference Services Quarterly, 20(1/2): 33-60.

Miles, Matthew B. and A. Michael Huberman. 1994. Qualitative Data Analysis: An Expanded Sourcebook. Thousand Oaks, CA: Sage.

Muller, Michael J. and Sarah Kuhn. 1993. "PD.” Communications of the ACM 36(6): 24-28

Patton, Michael Q. 2002. Qualitative research and evaluation methods, $3^{\text {rd }}$ Edition. Thousand Oaks, CA: Sage

Scaife, Michael and Yvonne Rogers. 1999. "Kids as informants: telling us what we didn't know or confirming what we knew already." Chapter 2 in The design of children's technology. Morgan San Francisco, CA: Kaufmann Publishers.

Soloway, Elliot, Mark Guzdial and Kenneth E. Hay. 1994. "Learner-centered design: The challenge for HCI in the $21^{\text {st }}$ century." Interactions 1(2): 36-48.

Wilson, Thomas D. 1980. Recent trends in user studies: action research and qualitative methods. Reprinted in Information Research, 2000, 5(3). Retrieved from: http://www.informationr.net/ir/53/paper76.html\#six 